\title{
Highly Tunable Electrostatic Nanomechanical Resonators
}

\author{
Syed N. R. Kazmi, ${ }^{\bar{\top}}$ Amal Z. Hajjaj, ${ }^{\bar{\top}}$ Md A. Al Hafiz, Pedro M. F. J. Costa, and Mohammad I. \\ Younis*
}

\begin{abstract}
There has been significant interest towards highly tunable resonators for on-demand frequency selection in modern communication systems. Here, we report highly tunable electrostatically actuated silicon-based nanomechanical resonators. In-plane doubly-clamped bridges, slightly curved as shallow arches due to residual stresses, are fabricated using standard electron beam lithography and surface nanomachining. The resonators are designed such that the effect of mid-plane stretching dominates the softening effect of the electrostatic force. This is achieved by controlling the gap-to-thickness ratio and by exploiting the initial curvature of the structure from fabrication. We demonstrate considerable increase in the resonance frequency of nanoresonators with the dc bias voltages up to $108 \%$ for $180 \mathrm{~nm}$ thick structures with a transduction gap of $1 \mu \mathrm{m}$ separating them from the driving/sensing electrodes. The experimental results are found in good agreement with those of a nonlinear analytical model based on the Euler-Bernoulli beam theory. As a potential application, we demonstrate a tunable narrow band-pass filter using two electrically coupled nanomechanical arch resonators with varied dc bias voltages.
\end{abstract}

Index Terms-Doubly-clamped bridges, electrostatic force, nanomechanical resonator, shallow arch, tunability.

\section{INTRODUCTION}

$\mathrm{T}$ UNABLE micro/nano electro mechanical systems (M/NEMS) resonators are highly desirable for on-demand frequency selection in modern communication systems [1]. Such resonators have been also proposed for numerous applications, such as mass/gas sensors [2,3], memory elements [4,5], logic devices [6,7], gyroscopes [8], energy harvesters [9], and signal processing elements [10,11]. Generally, the resonance frequency of a resonator can be tuned by changing its stiffness through applied axial loads, which can be applied by electrostatic [12], electromagnetic [13], or electrothermal [14] actuations. Recent studies have shown tunability of doubly-clamped microbeam resonators by axially controlling their stiffness electrostatically [12] and electrothermally [14].

The tunability of MEMS resonators using electrostatic forces

Manuscript received xxxxxx xx, 2017. This work was supported by funding from King Abdullah University of Science and Technology (KAUST) research grant. The review of this paper was arranged by xxxxxxxxxx.

S. N. R. Kazmi, A. Z. Hajjaj, M. M. Al Hafiz and M. I. Younis are with the Nano/Micro Mechanics and Motion Laboratory, Physical Science and Engineering Division, King Abdullah University of Science and Technology, Thuwal, 23955-6900, Saudi Arabia. has been the most used technique since the early work of Nathanson [15]. However, it suffers a major drawback due to the pull-in instability. Particularly high dc voltages can lead to the collapse of the resonating microstructures compromising the device and thus limiting its tunability [16]. Typically, the electrostatic force induces a softening effect in the straight doubly-clamped beams, resulting in a reduction of their resonance frequency [17]. A recent study however has demonstrated the possibility of increasing the resonance frequency while increasing the dc voltage when controlling the gap-to-thickness ratio [18].

At the nano scale, several studies have investigated the variation of the fundamental resonance frequency with the electrostatic voltage [19-23]. The tunability of the fundamental frequency has been demonstrated for slacked carbon nanotubes (CNTs) as varying the applied voltage [19-22]. Recently, the resonance frequency of a slacked single-walled CNT was controlled using an electrothermal actuator [24].

The fabrication of thin straight submicron and nanobeams is quite challenging due to the residual stresses and the thickness variation observed in the device layers. Thus, it is critical to understand the effects of such imperfections on the resonance frequency of nanobeams, and hence be able to utilize them. Here, we present an analytical and experimental study demonstrating high tunability of NEMS beams curved as shallow arches. The model considers variety of critical geometric parameters, mainly the thickness of the arch, its initial curvature, and the transduction gap between the beam and the stationary electrodes. These initially curved siliconbased structures may not compete with carbon nanotubes in terms of frequency tunability. However, the fabrication process of these resonators is controllable and simpler compared to the less controlled growth process of carbon nanotubes (the ability to control their curvature and dimensions), hence are less practical for frequency tunable devices. As shown, these structures can be used as the basic building blocks for tunable band-pass filters for high frequency applications.

P. M. F. J. Costa is with the Laboratory for Carbon Nanostructures, Physical Science and Engineering Division, King Abdullah University of Science and Technology, Thuwal, 23955-6900, Saudi Arabia.

(Corresponding author email: Mohammad.Younis@kaust.edu.sa).

Copyright (c) 2017 IEEE. Personal use of this material is permitted. However, permission to use this material for any other purposes must be obtained from the IEEE by sending a request to pubs-permissions@ieee.org. 


\section{MATERIAL AND METHODS}

\section{A. Problem Formulation}

The device under consideration, Fig. 1, consists of an initially curved doubly-clamped nanobeam, which is curved due to residual stresses from fabrication. This effect can be controlled to some extent through the choices of the beam length and thickness. The nanobeam is actuated by a dc polarization voltage $V_{d c}$ and an ac harmonic voltage of amplitude $V_{a c}$ and frequency $\Omega$, and is subjected to a viscous damping of coefficient $c$. The initial shape $w_{0}$ along the beam length $x$ is approximated to be of the buckled form [25] expressed expressed as

$w_{0}(x)=-\frac{1}{2} b_{0}\left(1-\cos \left(2 \pi \frac{x}{l}\right)\right)$

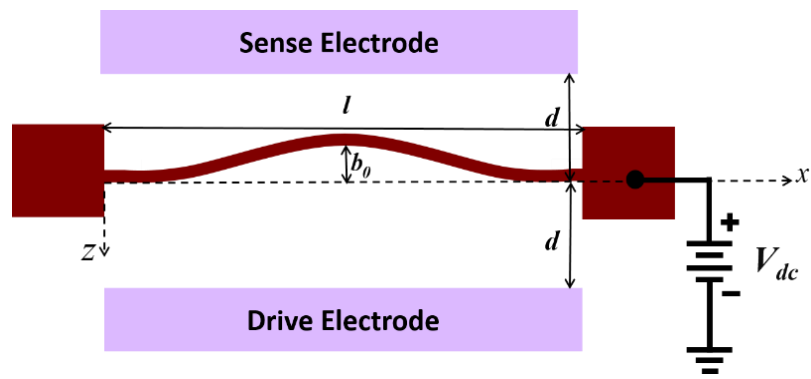

Fig. 1. Schematic of an electrostatically actuated doubly-clamped curved beam.

The nonlinear Euler-Bernoulli equation of motion governing the transverse deflection of the beam $w(x, t)$ in space and time $t$ is written as $[25,26]$ :

$$
\begin{aligned}
& \rho b h \frac{\partial^{2} w}{\partial t^{2}}+c \frac{\partial w}{\partial t}+E I_{s} \frac{\partial^{4} w}{\partial x^{4}}= \\
& \left(\frac{\partial^{2} w}{\partial x^{2}}+\frac{d^{2} w_{O}}{d x^{2}}\right)\left[N+\frac{E A}{2 l} \int_{O}^{l}\left(\left(\frac{\partial w}{\partial x}\right)^{2}+2 \frac{\partial w}{\partial x} \frac{d w_{0}}{d x}\right) d x\right] \\
& +\frac{1}{2} \varepsilon b \frac{\left(V_{d c}-V_{a c} \cos (\Omega t)\right)^{2}}{\left(d-w-w_{O}\right)^{2}}-\frac{1}{2} \varepsilon b \frac{V_{d c}}{\left(d+w+w_{O}\right)^{2}}
\end{aligned}
$$

The nanobeam is subjected to the fixed-fixed boundary conditions

$$
w(0, t)=w(l, t)=0 \text { and }\left.\frac{\partial w}{\partial x}\right|_{(0, t)}=\left.\frac{\partial w}{\partial x}\right|_{(l, t)}=0
$$

where $l, b$, and $h$ are the length, depth, and thickness of the beam, respectively, $d$ is the gap between the beam and the stationary electrodes, $\rho$ is the material density, $I_{s}=b h^{3} / 12$ is the moment of inertia of the cross section of area $A=b h, E$ is Young's modulus, and $\varepsilon$ is the dielectric constant of the gap medium. $N$ denotes the residual axial force originated from fabrication. The last term in equation (2) represents the electrostatic force from the sensing electrode.

In this work, a multi-mode Galerkin procedure is adopted to solve for the variation of the resonance frequency of the nanobeam with $V_{d c}$ [25]. To show more clearly the contribution of the various parameters on the resonance frequency, a one mode discretization is shown next, which provides a closed form approximate solution of the first resonance frequency as below

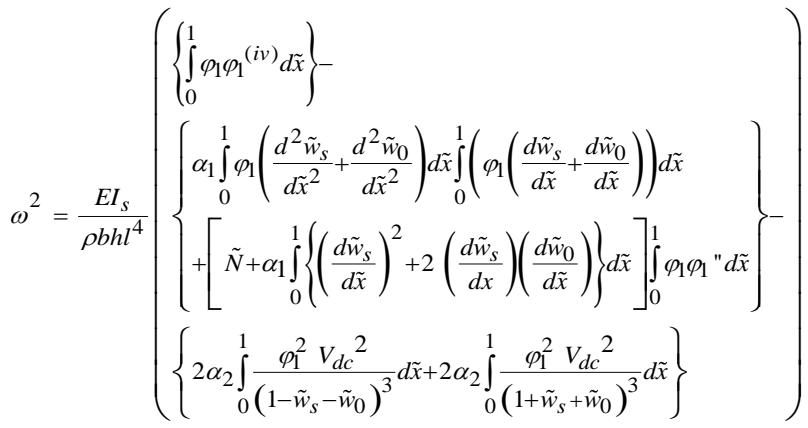

where

$$
\begin{gathered}
\tilde{x}=\frac{x}{l}, \tilde{w}_{0}=\frac{w_{0}}{d}, \tilde{w}_{s}=\frac{w_{s}}{d}, \alpha_{1}=6\left(\frac{d}{h}\right)^{2}, \alpha_{2}=\frac{6 \varepsilon l^{4}}{E h^{3} d^{3}}, \\
\tilde{N}=\frac{l^{2}}{E I_{s}} N
\end{gathered}
$$

where $\phi_{1}(x)$ denotes the first mode shape of the unactuated shallow arch, $\omega$ denotes the new natural frequency of the nanobeam under the dc bias voltage and $w_{s}(x)$ is the static deflection of the nanobeam due to $V_{d c}$ governed by

$$
\begin{aligned}
& E I_{s} \frac{d^{4} w_{s}}{d x^{4}}= \\
& \left(\frac{d^{2} w_{s}}{d x^{2}}+\frac{d^{2} w_{O}}{d x^{2}}\right)\left[N+\frac{E A}{2 l} \int_{0}^{l}\left(\left(\frac{d w_{S}}{d x}\right)^{2}+2 \frac{d w_{S}}{d x} \frac{d w_{O}}{d x}\right) d x\right] \\
& +\frac{1}{2} \varepsilon b \frac{V_{d c}^{2}}{\left(d-w_{s}-w_{O}\right)^{2}}-\frac{1}{2} \varepsilon b \frac{V_{d c}{ }^{2}}{\left(d+w_{s}+w_{O}\right)^{2}}
\end{aligned}
$$

The first term in the right hand side of equation (4) is positive and represents the bending contribution that implicitly describes the fundamental frequency of the nanobeam at zero $V_{d c}$. The second term is negative and represents the effect of the mid-plane stretching, axial load, and initial curvature. The last term is positive and represents the effect of the electrostatic force induced by the two-side electrodes. One can note that for a perfectly straight beam $w_{0}=0$ and $w_{S}=0$ (because of the two equal electrostatic forces of opposite signs), and thus the second term cancels out. Hence, the resonance frequency is only affected by the softening effect of the electrostatic force.

Equation (4) demonstrates that the eigenvalue problem, and hence the resonance frequency of the resonators, depends strongly on the thickness $h$, gap $d$, and the mid-beam initial rise $b_{0}$ (through $w_{0}$ ). It is possible to strengthen the stretching effect by minimizing $h$ and maximizing $d$, and hence maximizing $\alpha_{l}$, which tends to increase the resonance frequency to overcome the softening effect of the electrostatic force. Also note that the presence of $w_{0}$ is essential to create non-zero $w_{S}$, and hence induces mid-plane stretching. 
We study analytically the combined effect of the mid-beam initial rise and the gap-to-thickness ratio on the resonance frequency of silicon-based nanobeams. All the analytical results are obtained considering a Young's modulus of $169 \mathrm{GPa}$ and a Poisson's ratio of 0.27 for nanobeams of $15 \mu \mathrm{m}$ length, $850 \mathrm{~nm}$ depth, and $150 \mathrm{~nm}$ thickness. The results are obtained using five modes in the Galerkin procedure.

Figs. $2 \mathrm{a}-2 \mathrm{~d}$ show the variation of the beam midpoint deflection and the corresponding resonance frequency for various initial curvature rises for $250 \mathrm{~nm}$ and $850 \mathrm{~nm}$ transduction gaps between the beam and sensing/driving electrodes. For $b_{0}=0$, Fig. 2a indicates zero static deflection while Fig. 2b shows a continuous decrease in the resonance frequency. A slight increase in $b_{0}$ induces mid-plane stretching and curvature effects, which impact considerably the static deformation and hence the resonance frequency. For the cases of $850 \mathrm{~nm}$ gap, Figs. $2 \mathrm{a}$ and $2 \mathrm{~b}$, increasing the voltage always increases the curvature of the already curved beams resulting into continuous increase in the resonance frequency until finally reaching pull-in. Because of the relatively large gap compared to the thickness (gap-to-thickness ratio $=5.67$ ) and initial rise, the increase in stiffness always dominates the electrostatic force effect, despite the fact that the beam deforms toward one of the electrodes as increasing $V_{d c}$. For the cases of $250 \mathrm{~nm}$ gap, Figs. $2 \mathrm{c}$ and $2 \mathrm{~d}$, for small values of $b_{0}$ increasing $V_{d c}$ increases the curvature and the resonance frequency until pull-in, similar to the previous cases, in which the electrostatic force is dominated by the mid-plane stretching and curvature effects. For larges values of $b_{0}$, the beam is curved toward and very close to one of the electrostatic electrode. This maximizes the electrostatic force effect that dominates the curvature and stretching effects. This can be also noticed from the small gap-to-thickness ratio of 1.67 .

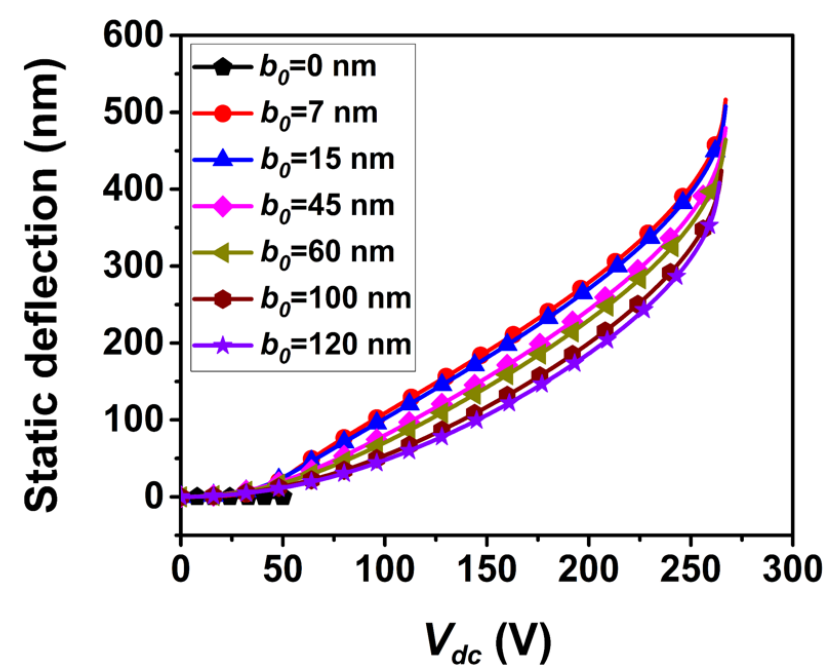

(a)

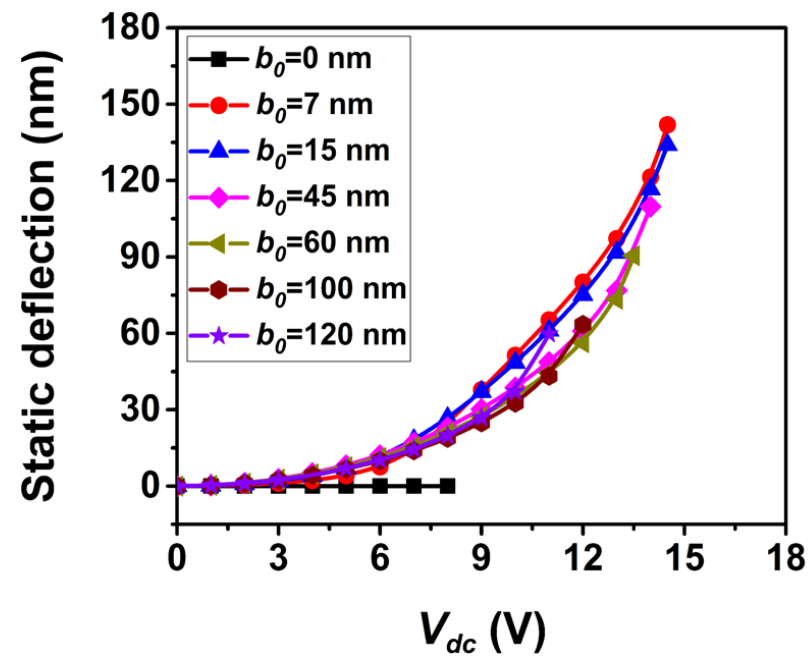

(c)

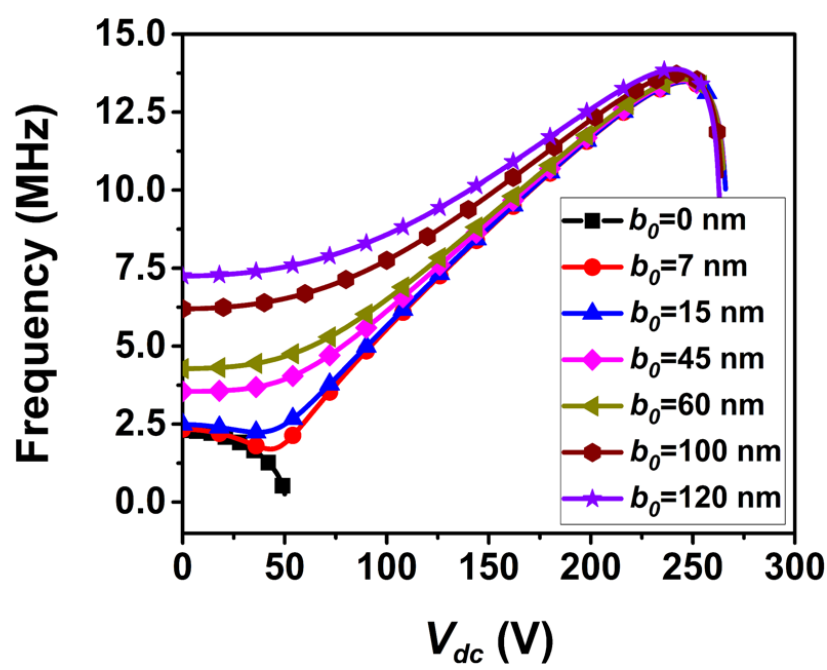

(b)

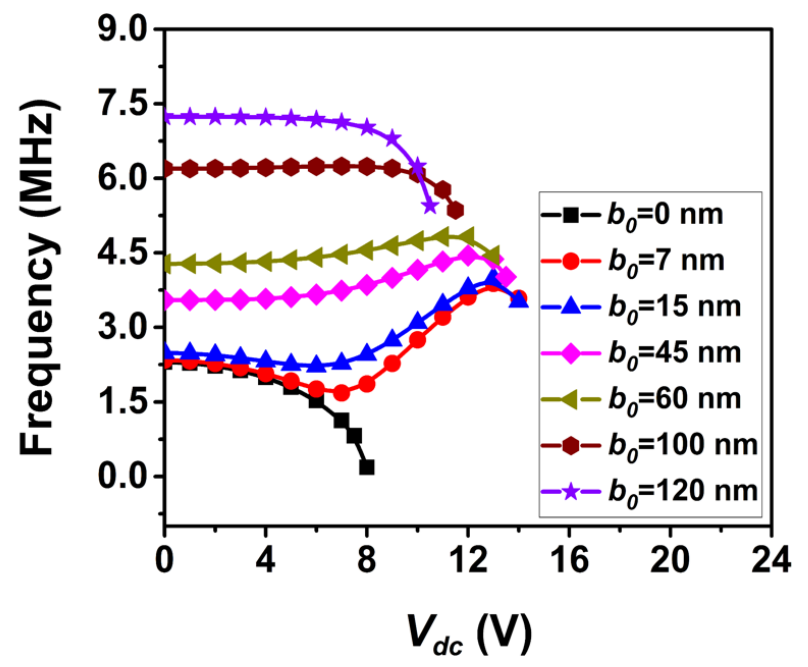

(d)

Fig. 2. The simulated static deflection of the beam midpoint and resonance frequency for various initial rises versus the dc bias voltages for initially curved nanobeams of $15 \mu \mathrm{m}$ length, $850 \mathrm{~nm}$ depth, $150 \mathrm{~nm}$ thickness, and the transduction gaps of $d=850 \mathrm{~nm}(\mathrm{a}, \mathrm{b})$ and $d=250 \mathrm{~nm}(\mathrm{c}, \mathrm{d})$. 
Next, we investigate the effect of the gap $d$ considering a similar nanobeam of Fig. 2 while fixing the curvature. Figs $3 \mathrm{a}$ and $3 \mathrm{~b}$ show the variation of the resonance frequency with $d$. Fig. 3a shows that for $d=150 \mathrm{~nm}$ (gap-to-thickness ratio=1) the softening effect due to electrostatic force is dominant over the mid-plane stretching; thus resulting in a decrease in the resonance frequency with increasing $V_{d c}$. On the other hand, for $d=250-450 \mathrm{~nm}$ (gap-to-thickness ratio=1.67-3) the frequency increases with the voltages, showing the dominant effect of mid-plane stretching over the softening effect of the electrostatic force. However, the tunability is still limited due to the relatively small pull-in voltages. For beams with larger transduction gaps, Fig. 3b, higher tunability ranges are achieved. This is clearly attributed to the dominant mid-plane

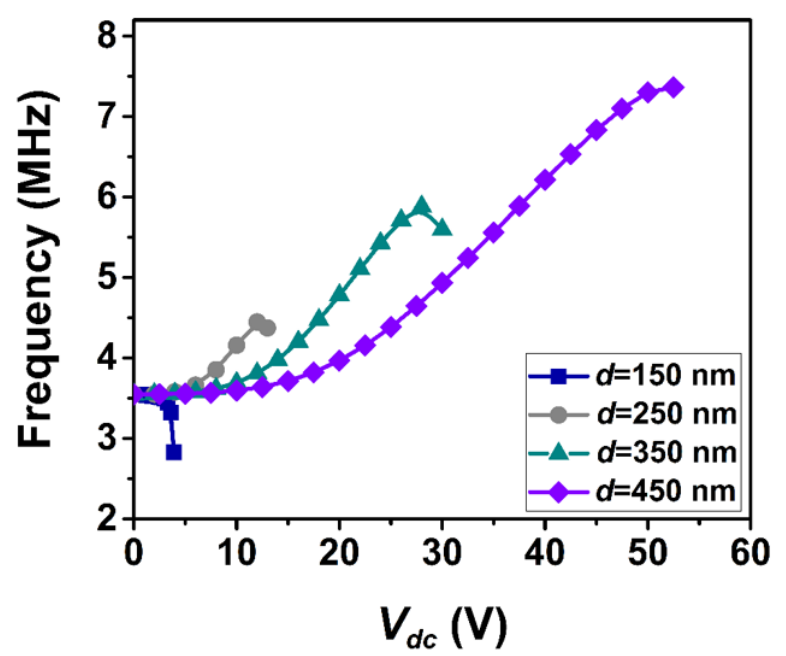

(a)

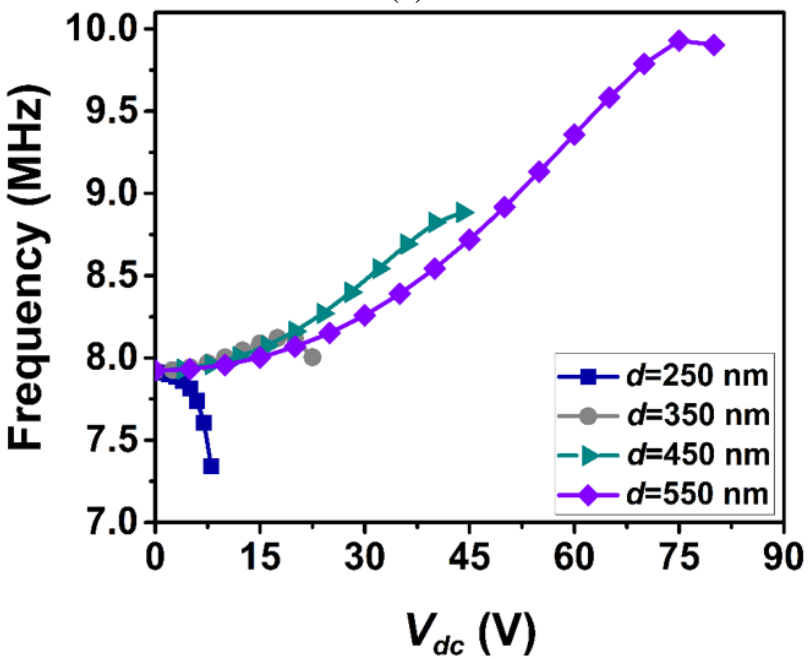

(c) stretching effect as indicated from the larger gap-to-thickness ratio and the initial curvature over the softening effect of the electrostatic force. Figs. $3 \mathrm{c}$ and $3 \mathrm{~d}$ show similar results for another case study of a beam with higher initial curvature of $b_{0}=130 \mathrm{~nm}$. The results indicate similar trend to that of Figs. 3a and $3 \mathrm{~b}$ however with higher tunability range. Therefore, a larger transduction gap compared to the thickness of the beam ensures dominant mid-plane stretching effect over the softening effect due to the electrostatic force, which results in frequency tuning towards higher frequency values. Also, a higher initial rise further adds to high frequency tunability due to the increased stiffness. Moreover, for large values of $\mathrm{d}$, very high pull-in voltages are predicted, which practically eliminate the possibility of pull-in.

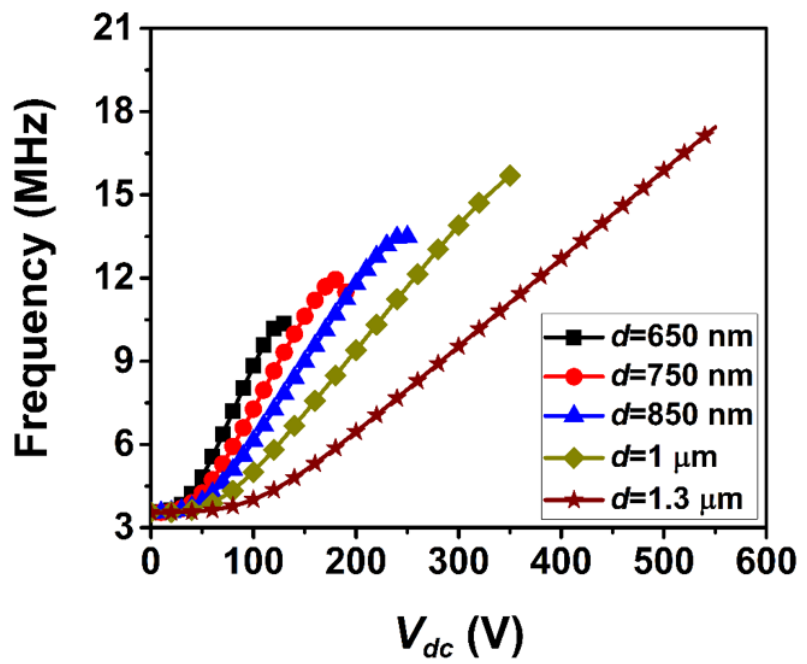

(b)

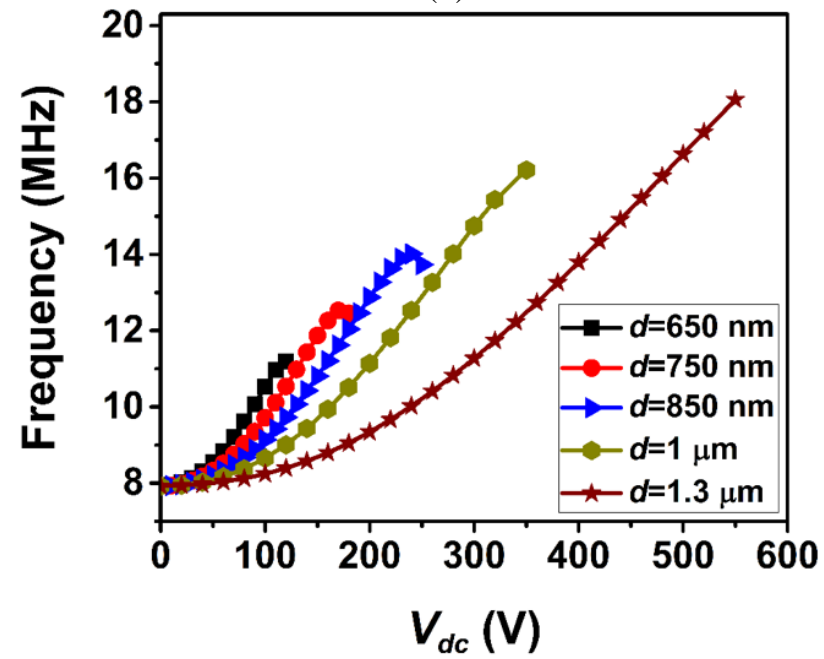

(d)

Fig. 3. The simulated resonance frequency versus dc voltage for beams of $15 \mu \mathrm{m}$ length, $850 \mathrm{~nm}$ depth, $150 \mathrm{~nm}$ thickness, (a,b) for $45 \mathrm{~nm}$ initial rise for various values of gap of (a) $d=150-450 \mathrm{~nm}$ and (b) $d=0.65-1.3 \mu \mathrm{m}$, and (c,d) for $130 \mathrm{~nm}$ initial rise for various values of gap of (c) $d=250-550 \mathrm{~nm}$ and (d) $d=0.65-1.3 \mu \mathrm{m}$.

\section{B. Device Fabrication}

To demonstrate the frequency tunability under the dc bias, doubly-clamped beams are fabricated from a $<100\rangle$ highlyconductive boron doped Si device layer of silicon-on-insulator (SOI) wafer with gap-to-thickness ratio of more than four. The curved nanobeams can be fabricated either by direct patterning of nanobeams with a defined initial rise on the nearly stress free highly doped device layer of SOI wafer or by patterning straight nanobeams on stressed highly doped device layer of SOI wafer 
that result in curved nanobeams upon release due to the residual stresses. The curvature of the nanobeams for a particular length is highly dependent on the residual stresses in the device layer. The residual stresses, and therefore the initial curvature, can be generated and controlled by hydrogen implantation of the device layer and later annealing it at high temperature, see ref [27]. The SOI wafer from Semiconductor Wafer Inc. (SWI) with residually stressed device layer is used to fabricate the shallow arches. Table I summarizes the dimensions of fabricated nanobeams. The fabrication process employs standard e-beam lithography and surface nanomachining techniques. The main fabrication steps are illustrated in Fig. 4. The fabrication starts with a standard cleaning of SOI wafer by dipping it in a heated piranha solution at $120^{\circ} \mathrm{C}$, followed by a deionized (DI) water rinse to remove the organic residues. The native oxide is removed by using $1 \% \mathrm{HF}$ solution until the surface turns to hydrophobic followed by DI water rinse and spin drying, Fig. 4a. The thickness of the device layer is reduced from $2 \mu \mathrm{m}$ to $850 \mathrm{~nm}$ by thermal oxidation of the entire SOI wafer, Fig. $4 \mathrm{~b}$ and then removing this thermally grown oxide using buffered oxide etch (BOE) solution at room temperature, Fig. 4c. The wafer is than diced into $1.2 \mathrm{~cm} \times 1.2 \mathrm{~cm}$ samples for further processing after standard cleaning as described earlier. A uniform layer of $\sim 210 \mathrm{~nm}$ positive tone e-beam lithography (EBL) resist 950 PMMA A4 is spun on the chip to transfer the desired pattern on the device layer, Fig. 4d. EBL is performed at low electron beam current of $\sim 500 \mathrm{pA}$ to achieve dimensions in nanometers range. The resist is then developed in a solution of MIBK and IPA (1:3) for $90 \mathrm{~s}$ followed by a deionized water rinse Fig. 4e. Reactive ion etching (RIE) recipe consisting of alternate cycles of $\mathrm{C}_{4} \mathrm{~F}_{8}$ and $\mathrm{SF}_{6}$ is used to separate the nanobeam, anchoring pads, and the RF electrodes from the rest of wafer surface, Fig. 4f. This entire wafer surface will later serve as ground while characterizing the fabricated devices. The EBL resist is then dissolved using heated acetone at $55{ }^{\circ} \mathrm{C}$ to have a clean device surface for probing the dc, sensing/driving electrodes and the ground plane, Fig. 4g. Finally, the HF vapor etch is performed to have free standing nanobeams by removing the underneath $\sim 1 \mu \mathrm{m}$ box layer, Fig. $4 \mathrm{~h}$.

\begin{tabular}{|c|c|c|c|c|}
\hline & $\begin{array}{c}\text { Length } \\
(\boldsymbol{\mu} \mathrm{m})\end{array}$ & $\begin{array}{c}\text { depth } \\
(\mathrm{nm})\end{array}$ & $\begin{array}{c}\text { Thickness } \\
(\mathrm{nm})\end{array}$ & $\begin{array}{c}\text { Gap } \\
(\boldsymbol{\mu m})\end{array}$ \\
\hline D1 & 15 & 850 & 130 & 0.85 \\
\hline D2 & 15 & 850 & 130 & 1 \\
\hline D3 & 15 & 850 & 180 & 0.85 \\
\hline D4 & 15 & 850 & 180 & 1 \\
\hline
\end{tabular}

Table I. Designed dimensions for the fabricated nanobeams.

Fig. 5 shows an SEM image of a curved beam fabricated using the fabrication process flow of Fig. 4. Note that the beam is slightly curved due to the residual stress in the device layer. Therefore, thinner beams are prone to relatively higher initial curvature than the thicker beams after the HF vapor release. The observed initial rise is found between $\sim 100 \mathrm{~nm}$ to $\sim 150 \mathrm{~nm}$ for beams of thicknesses $130 \mathrm{~nm}$ and $180 \mathrm{~nm}$.

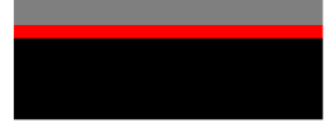

Substrate preparation

(a)

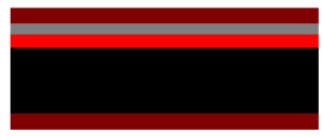

Thermal oxidation

(b)

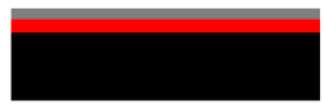

Buffered oxide etching

(c)

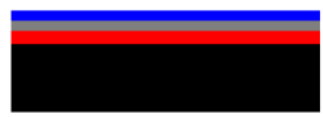

PMMA resist spinning

(d)

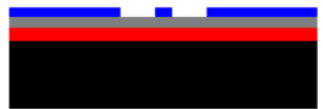

PMMA resist patterning

(e)

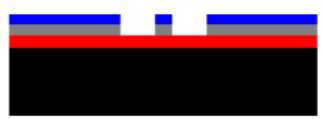

RIE of device layer

(f)

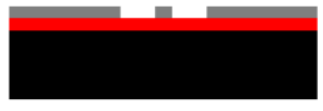

Resist removal

(g)

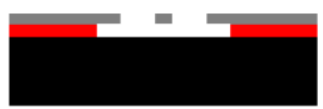

HF vapor etch

(h)

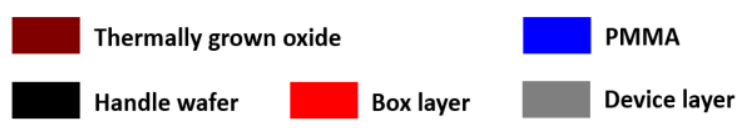

Fig. 4. Process flow for the fabrication of the nanobeams. (a) Substrate cleaning and native oxide removal. (b) Thermal oxidation to thin down the device layer. (c) BOE treatment to remove the thermally grown oxide. (d) Spinning of ebeam resist (950 PMMA A4) to achieve $\sim 210 \mathrm{~nm}$ thickness. (e) Patterning of 950 PMMA A4. (f) RIE to fabricate the nanobeams and to separate the entire device structure from the ground plane. (g) E-beam resist removal in heated acetone. (h) HF vapor etching for free standing nanobeams.

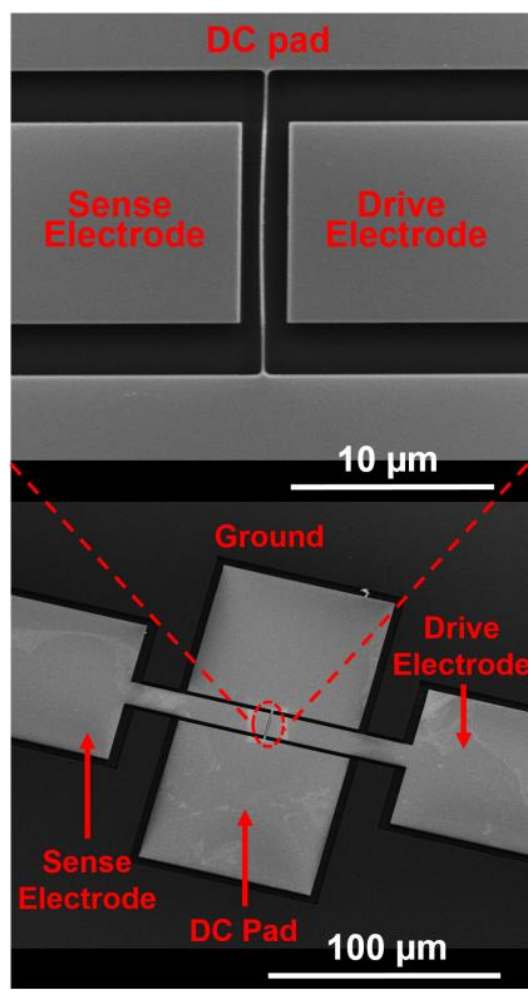

Fig. 5. An SEM image of the entire device structure (bottom) along with a magnified view (top) showing the nanobeam and the transduction gap separating it from the driving/sensing electrodes. 


\section{Measurements}

The electrical characterization is performed in an ST-500 JANIS probe station. Fig. 6 shows a schematic of the driving and sensing configuration of the beam in its two-port measurement configuration. The beam is biased by a dc bias voltage and driven at resonance by an ac excitation signal provided at the driving electrode from one of the ports of the network analyzer (Agilent E5071C). The motional current is capacitively sensed through the other electrode, named as sensing electrode, and is fed into a low-noise amplifier (LNA) with its output coupled to the other port of the network analyzer. All the experiments are performed at $5.2 \times 10^{-5}$ mbar pressure and at room temperature.

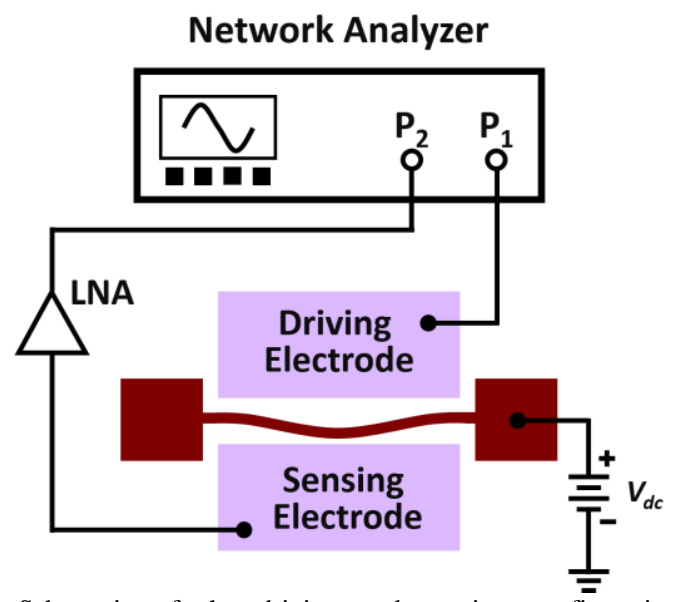

Fig. 6. Schematic of the driving and sensing configuration of the nanomechanical shallow arch beams.

Fig. 7 shows frequency responses under various applied dc bias conditions for the D1 beam. This shows an increase in the natural frequency of the beam with an increase in the dc polarization voltage attributed to the dominant mid-plane stretching effect over softening effect of electrostatic force. Note that the drive power is adjusted accordingly to have linear frequency response beyond $40 \mathrm{~V}$.

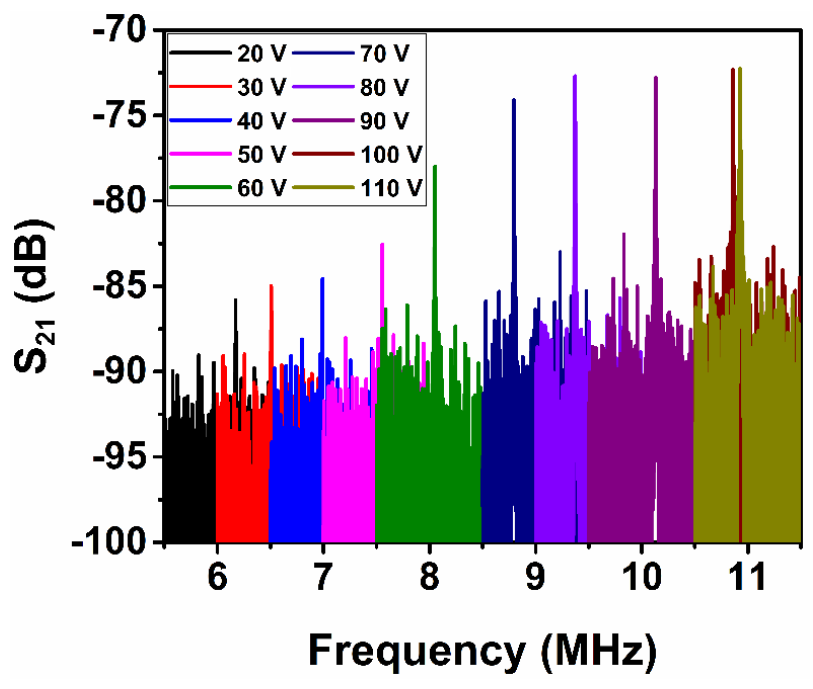

Fig. 7. The measured frequency response for varied dc voltages for beam of 15 $\mu \mathrm{m}$ length, $850 \mathrm{~nm}$ depth, $130 \mathrm{~nm}$ thickness and a transduction gap of $850 \mathrm{~nm}$.

\section{RESULTS AND DISCUSSIONS}

Fig. $8 \mathrm{a}$ and Fig. $8 \mathrm{~b}$ show the variation of the resonance frequency of the $130 \mathrm{~nm}$ and $180 \mathrm{~nm}$ thick nanobeams of $15 \mu \mathrm{m}$ length, $850 \mathrm{~nm}$ depth, and with the initially designed transduction gaps of $850 \mathrm{~nm}$ and $1 \mu \mathrm{m}$, respectively, between the nanobeam and the driving/sensing electrodes for various $\mathrm{dc}$ polarization voltages. The figures compare the analytical and experimental data. The analytical results here are obtained by considering the dimensions of Table II, which indicates thickness values different from the nominal designed ones due to the fabrication imperfections and tolerances.

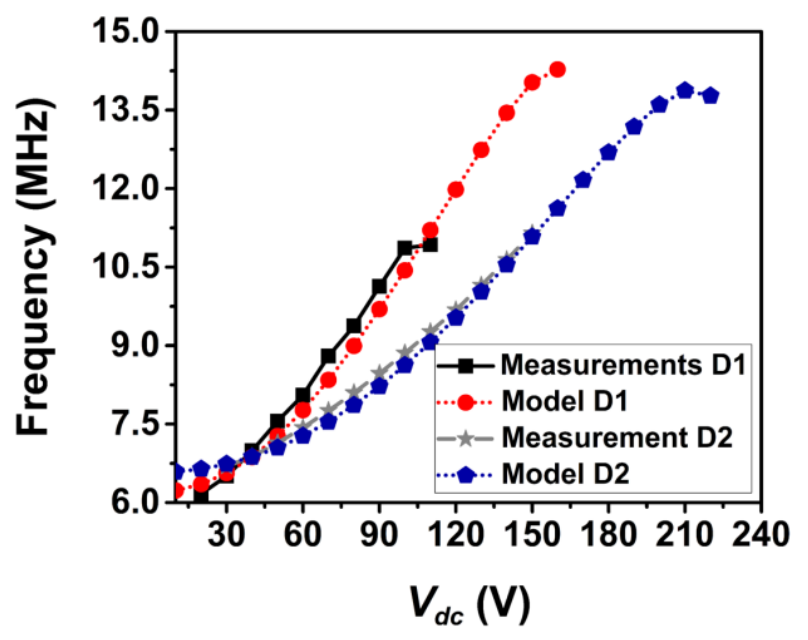

(a)

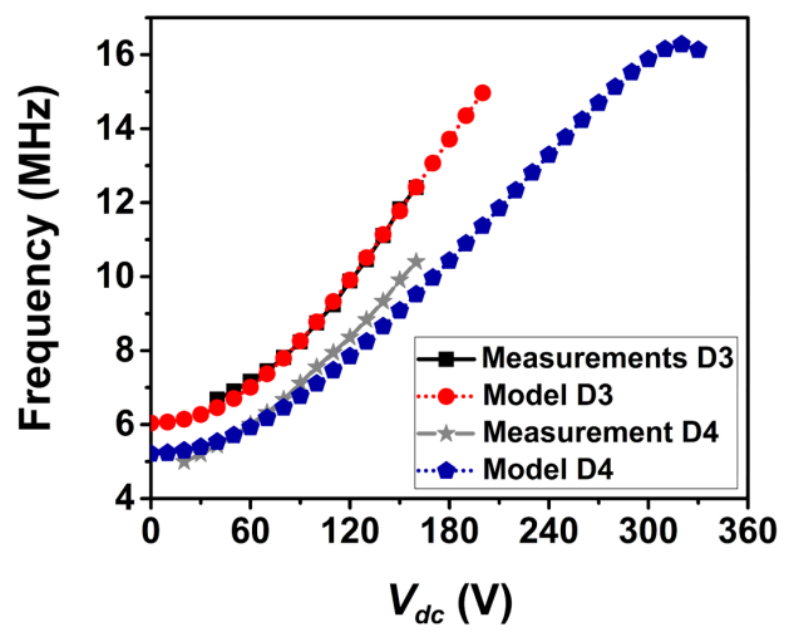

(b)

Fig. 8. The resonance frequency versus dc voltage for beams of $15 \mu \mathrm{m}$ length, $850 \mathrm{~nm}$ depth, and (a) $130 \mathrm{~nm}$ thickness with a transduction gap of $850 \mathrm{~nm}$ and $1 \mu \mathrm{m}$, respectively, (b) $180 \mathrm{~nm}$ thickness with a transduction gap of $850 \mathrm{~nm}$ and $1 \mu \mathrm{m}$, respectively. In (a) and (b), simulations results are compared with the experimental data.

\begin{tabular}{|c|c|c|c|c|c|}
\hline & $\begin{array}{c}\text { Length } \\
(\boldsymbol{\mu} \mathbf{m})\end{array}$ & $\begin{array}{c}\text { depth } \\
(\mathbf{n m})\end{array}$ & $\begin{array}{c}\text { Thickness } \\
(\mathbf{n m})\end{array}$ & $\begin{array}{c}\text { Gap } \\
(\mathbf{n m})\end{array}$ & $\begin{array}{c}\text { Initial rise } \\
(\mathbf{n m})\end{array}$ \\
\hline D1 & 15 & 850 & 70 & 850 & 122 \\
\hline D2 & 15 & 850 & 120 & 850 & 116 \\
\hline D3 & 15 & 850 & 70 & 1000 & 123 \\
\hline D4 & 15 & 850 & 120 & 1000 & 97.3 \\
\hline
\end{tabular}

Table II. Dimensions used for obtaining the analytical results. 
The results show an increase in the resonance frequency for the beams with the increased dc bias voltages. The beams of $130 \mathrm{~nm}$ thickness with $850 \mathrm{~nm}$ and $1 \mu \mathrm{m}$ transduction gap between the beam and the driving/sensing electrodes show tunability of $77.12 \%$ and $85.51 \%$ under dc biased conditions, respectively. Similarly, the resonance frequencies of arches of $180 \mathrm{~nm}$ thickness are also found to increase with increasing the dc polarization voltages with tunability of $73.05 \%$ and $108.14 \%$ for $850 \mathrm{~nm}$ and $1 \mu \mathrm{m}$ transduction gap between the beam and the driving/sensing electrodes, respectively. A closer look towards the tunability of these beams reveals that those with 1 $\mu \mathrm{m}$ transduction gaps have higher $\%$ tunability, i.e., $66.07 \%$ and $45.14 \%$ compared to similar beams with $850 \mathrm{~nm}$ transduction gaps, i.e., $56.29 \%$ and $34.71 \%$ for dc bias changes from $40 \mathrm{~V}$ to $110 \mathrm{~V}$, respectively. This is clearly attributed to the dominating combined effect of thickness to gap ratio and the presence of initial curvature of the nanobeam over the softening effect of the electrostatic force for large gaps. Moreover, thicker arches can achieve higher tunability compared to the thin arches due to the relatively higher pull-in voltages. However, the thinner beams show higher tunability per unit increase in the dc polarization voltage due to the combined effect of increasing arch stiffness and dominating effect of mid-plane stretching over electrostatic force for large gaps.

Next, we demonstrate a proof of concept narrow band-pass filter using such tunable beams. Fig. 9a shows a schematic of the measurement setup for two near-identical electrically coupled resonators for narrow band-pass filter demonstration. Coupled resonators for filtering applications have been also demonstrated in [28-30]. The two resonators are biased in such a way that the first dc source results in equal voltage on the beams. A second dc source is used to tune the resonance frequency of the resonators with lower resonance frequency and to bring it closer to the resonance frequency of other resonator. This slight difference in resonance frequency of the two resonators is due to fabrication tolerances. The input RF signal from port 1 of the network analyzer is fed into the input electrodes of the two resonators through a power splitter. The output is received at a common sensing electrode and directly fed into the LNA with its output coupled to the other port of the network analyzer. Fig. 9b shows an SEM image of the fabricated device for band-pass filtering along with a magnified view of one of the arch resonators.

Fig. 10a shows the magnitude of the $S_{21}$ transmission parameters (response in black) for the electrically coupled resonators indicating resonance peaks at $5.186 \mathrm{MHz}$ and $5.147 \mathrm{MHz}$ for a dc bias voltage of $40 \mathrm{~V}$ and input RF power of $3.3 \mathrm{dBm}$ from port 1 of the network analyzer. Resonator 2 is tuned by applying an additional dc voltage so that both resonance peaks come closer (response in gray) to realize a narrow band-pass filter, which has a center frequency of $5.175 \mathrm{MHz}$ and a $3 \mathrm{~dB}$ band-width of $\sim 33 \mathrm{kHz}$, Fig. 10b. The filter's center frequency can be tuned to $5.446 \mathrm{MHz}$ with a $3 \mathrm{~dB}$ band-width of $\sim 19 \mathrm{kHz}$ (response in blue) by increasing the dc bias voltage to $50 \mathrm{~V}$ and tuning the frequency of the second resonator using second dc source to achieve narrow band filter response, Fig. 10b. The insertion loss of $-85 \mathrm{~dB}$ for the demonstrated bandpass filter is still quite high. This can be reduced by adding low noise amplifier (LNA) as conventionally done in resonator based band pass filtering [1]. The practical realization of tunable band-pass filters using electrically coupled nanomechanical arches with the flexibility of adjustable band-width can open up the possibilities for High Frequency (HF) filtering applications. Very Higher Frequency (VHF) filtering can be achieved by shrinking the device dimensions for similar designs using materials with higher Young's modulus to density ratio compared to silicon, such as silicon carbide [31].

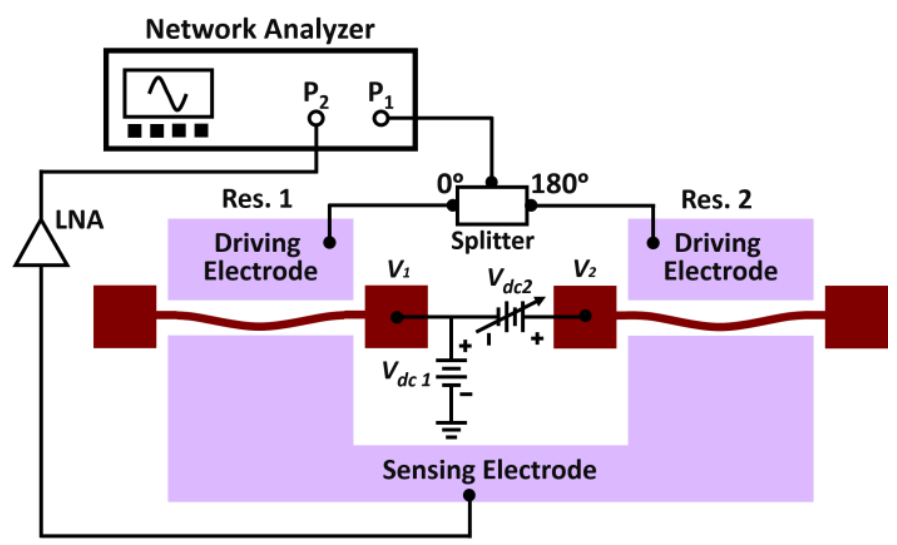

(a)

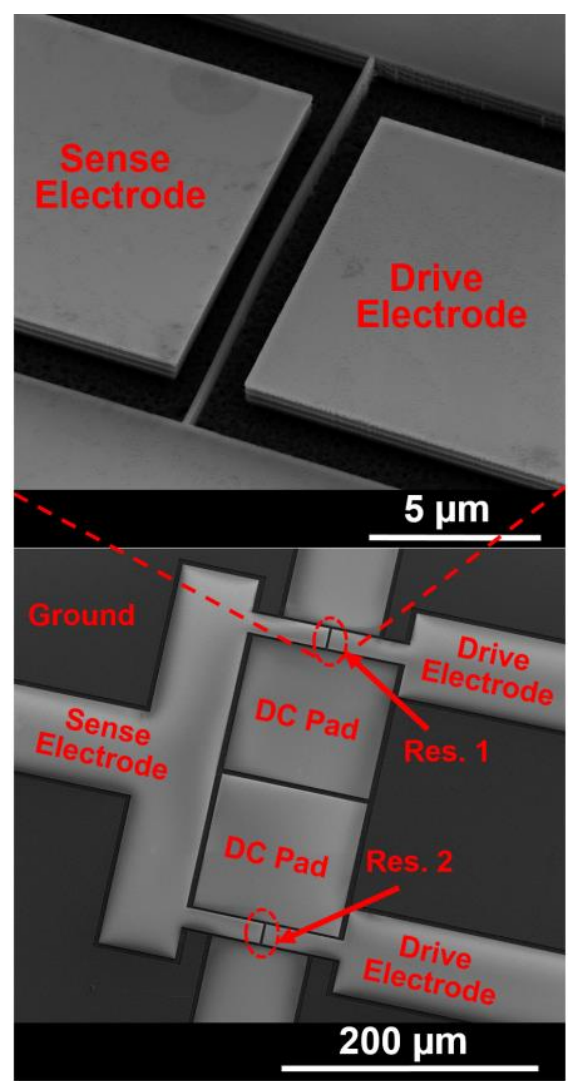

(b)

Fig. 9. (a) Schematic of the driving and sensing configuration for the electrically coupled nanomechanical arch beams under dc bias conditions for narrow bandpass filtering. (b) An SEM image showing the layout for the electrically coupled beams (bottom) for filtering along with a magnified view (top) showing the nanobeam of $15 \mu \mathrm{m}$ length, $850 \mathrm{~nm}$ depth, $180 \mathrm{~nm}$ thickness, and $1 \mu \mathrm{m}$ transduction gap between the beam and the driving/sensing electrodes. 


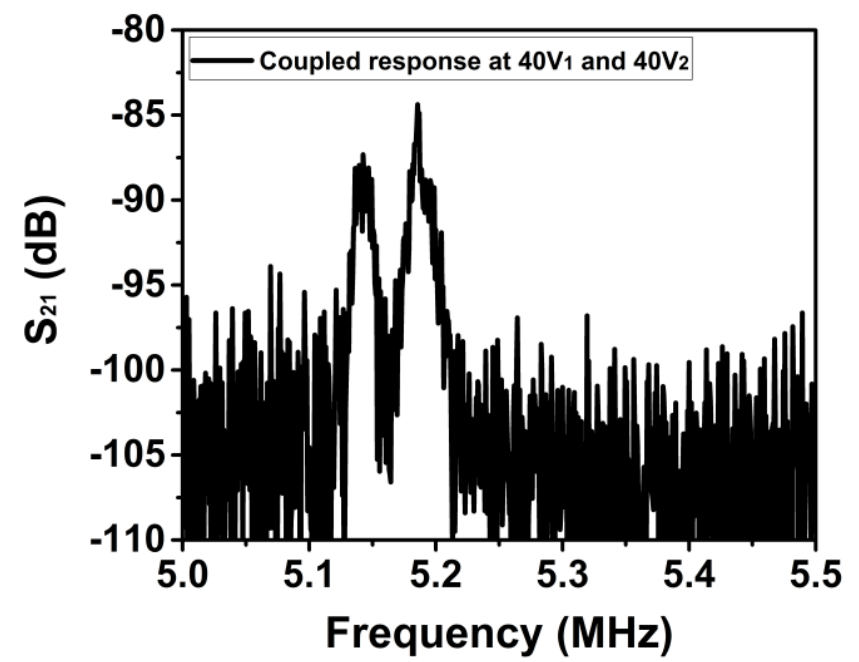

(a)

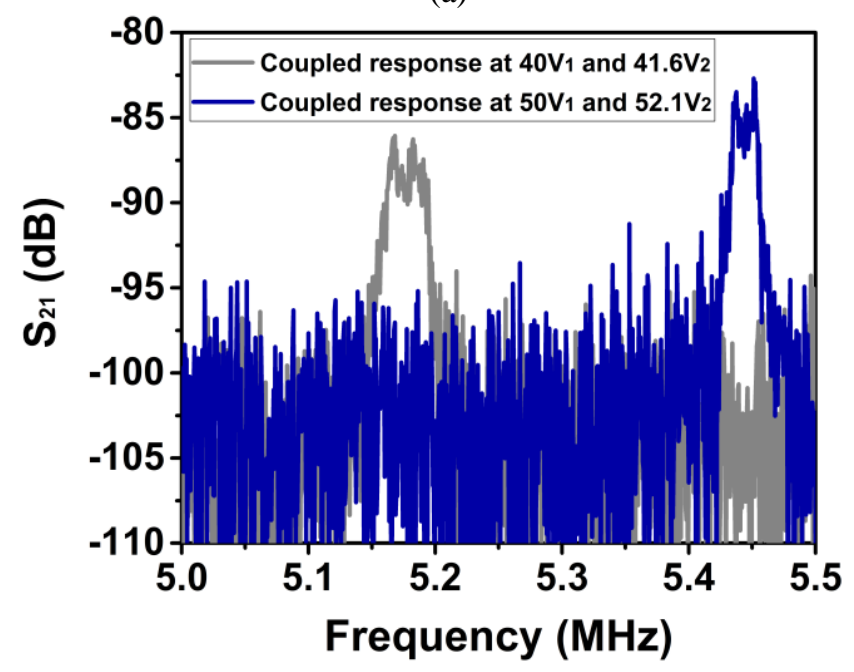

(b)

Fig. 10. (a) The magnitude of $S_{21}$ transmission parameter for two electrically coupled nanobeams at dc bias of $40 \mathrm{~V}$ and $3.3 \mathrm{dBm}$ RF power with quality factors of 370 and 382, respectively. (b) The magnitude of $S_{21}$ transmission parameter for the electrically coupled beams demonstrating a tunable narrow band-pass filter in high frequency range under dc polarization voltages of $40 V_{I}$ and $50 V_{1}$ (on arch 1) and $41.6 V_{2}$ and $52.1 V_{2}$ (on arch 2) at $3.3 \mathrm{dBm}$ input RF power.

Note here that the proposed device requires low vacuum environment to enable efficient excitation of the beam in resonance. The pressure requirements for this class of tunable nanoresonators for the targeted applications can be easily met while encapsulating them at the wafer scale using thin film encapsulation technique [32].

A final note regarding this filter is related to the power handling capability of the resonators, which depends on the linear operation range of the resonators while increasing the input power. To assess this, we have performed measurements, Fig. 11, on another similar resonator. We have experimentally found that these resonators undergoes nonlinear behavior for input power beyond $2 \mathrm{dBm}$ which limits their functionality as filters at high power levels exceeding $2 \mathrm{dBm}$.

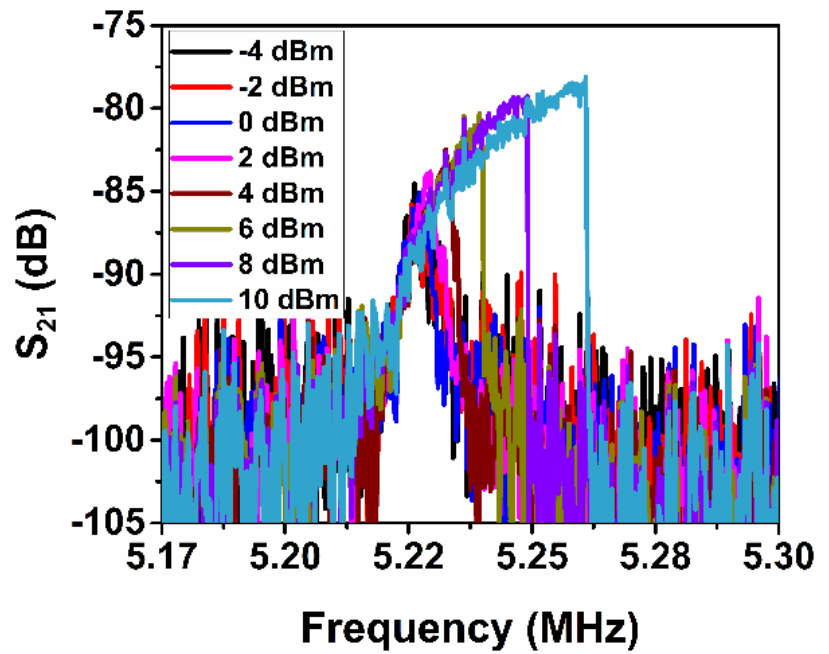

Fig. 11. The measured frequency response at $40 \mathrm{~V}$ for varied input RF power for beam of $15 \mu \mathrm{m}$ length, $850 \mathrm{~nm}$ depth, $180 \mathrm{~nm}$ thickness and a transduction gap of $1000 \mathrm{~nm}$.

\section{CONCLUSION}

An analytical and experimental study has been presented on the tunability of in-plane doubly-clamped nanomechanical beams curved as shallow arches under varied dc bias conditions. Through careful designs of the gap-to-thickness ratios and by exploiting the induced axial residual stresses to yield slightly curved structures, significant tuning ranges have been presented. Good agreement has been shown among the analytical and experimental data. Overall, the beams have shown high tunability by electrostatic actuation due to the dominant mid-plane stretching effect over the softening effect exerted by the electrostatic force. We interpret that this midplane stretching effect arises due to the combined effect of the gap-to-thickness ratio and the initial curvature of the nanobeams. Experimentally, an increase of the resonance frequency by $108.14 \%$ for some of the nanobeams is reported. It is demonstrated that the curvature is essential to reach such tunability and to increase the pull-in voltage. It is concluded that the observed large tunability is a result of two combined effects of the proper curvature and the large gap-thickness ratio. Finally, a proof-of-concept tunable narrow band-pass filter is also demonstrated using the tunable nanobeams. The high tunability of such beams may open up the possibilities for tunable band-pass filtering in the high frequency range (3-30 $\mathrm{MHz}$ ).

\section{ACKNOWLEDGEMENTS}

The authors would like to thank Nitin Batra for his help with the measurement setup. This work was supported by King Abdullah University of Science and Technology (KAUST) research funds.

\section{AUTHOR CONTRIBUTIONS}

${ }^{\bar{T}}$ Syed N. R. Kazmi and Amal Z. Hajjaj contributed equally to this work. 


\section{REFERENCES}

[1] C. T.-C. Nguyen, "Frequency-selective MEMS for miniaturized low-power communication devices", IEEE Trans. Microw. Theory Tech., vol. 47, pp. 1486-1503, 1999.

[2] E. Sage, A. Brenac, T. Alava, R. Morel, C. Dupré, M. S. Hanay, et al., "Neutral particle mass spectrometry with nanomechanical systems", Nat. Commun., vol. 6, 2015.

[3] I. Bargatin, E. Myers, J. Aldridge, C. Marcoux, P. Brianceau, L. Duraffourg, et al., "Large-scale integration of nanoelectromechanical systems for gas sensing applications", Nano Lett., vol. 12, pp. 1269-1274, 2012.

[4] T. Rueckes, K. Kim, E. Joselevich, G. Y. Tseng, C.-L. Cheung, and C. M. Lieber, "Carbon nanotube-based nonvolatile random access memory for molecular computing", Science, vol. 289, pp. 94-97, 2000.

[5] M. A. Al Hafiz, L. Kosuru, and M. I. Younis, "Electrothermal Frequency Modulated Resonator for Mechanical Memory", J. Microelectromech. Syst., vol. 25, pp. 877-883, 2016.

[6] S. N. Kazmi, M. A. Hafiz, K. N. Chappanda, S. Ilyas, J. Holguin, P. M. Costa, et al., "Tunable nanoelectromechanical resonator for logic computations", Nanoscale, vol. 9, pp. 34493457, 2017.

[7] M. Hafiz, L. Kosuru, and M. I. Younis, "Microelectromechanical reprogrammable logic device", Nat. Commun., vol. 7, 2016.

[8] H. Xie and G. K. Fedder, "Fabrication, characterization, and analysis of a DRIE CMOS-MEMS gyroscope", IEEE Sensors J., vol. 3, pp. 622-631, 2003.

[9] Q. Shi, T. Wang, and C. Lee, "MEMS based broadband piezoelectric ultrasonic energy harvester (PUEH) for enabling self-powered implantable biomedical devices", Sci. Rep., vol. $6,2016$.

[10] L. Lin, R. T. Howe, and A. P. Pisano, "Microelectromechanical filters for signal processing", $J$. Microelectromech. Syst., vol. 7, pp. 286-294, 1998.

[11] H. Chandrahalim, D. Weinstein, L. F. Cheow, S. A Bhave, "High- $\kappa$ dielectrically transduced MEMS thickness shear mode resonators and tunable channel-select RF filters", Sens. Actuators A Phys., vol. 136, pp. 527-539, 2007.

[12] E. M. Abdel-Rahman, M. I. Younis, and A. H. Nayfeh, "Characterization of the mechanical behavior of an electrically actuated microbeam", J. Micromech. Microeng., vol. 12, pp. 759, 2002.

[13] H. Maekoba, P. Helin, G. Reyne, T. Bourouina, and H. Fujita, "Self-aligned vertical mirror and V-grooves applied to an optical-switch: modeling and optimization of bi-stable operation by electromagnetic actuation", Sens. Actuators A Phys., vol. 87, pp. 172-178, 2001.

[14] A. Z. Hajjaj, N. Alcheikh, A. Ramini, M. A. Al Hafiz, and M. I. Younis, "Highly tunable electrothermally and electrostatically actuated resonators", J. Microelectromech. Syst., vol. 25, pp. 440-449, 2016.

[15] H. C. Nathanson, W. E. Newell, R. A. Wickstrom, and J. R. Davis, "The resonant gate transistor", IEEE Trans. Electron Dev., vol. 14, pp. 117-133, 1967.

[16] M. I. Younis, R. Miles, and D. Jordy, "Investigation of the response of microstructures under the combined effect of mechanical shock and electrostatic forces", J. Micromech. Microeng., vol. 16, pp. 2463, 2006.

[17] A. H. Nayfeh, M. I. Younis, and E. M. Abdel-Rahman, "Reduced-order models for MEMS applications", Nonlinear Dyn., vol. 41, pp. 211-236, 2005.

[18] A. Z. Hajjaj, A. Ramini, and M. I. Younis, "Experimental and analytical study of highly tunable electrostatically actuated resonant beams", J. Micromech. Microeng., vol. 25, pp. $125015,2015$.

[19] V. Sazonova, Y. Yaish, H. Üstünel, D. Roundy, T. A. Arias, and P. L. McEuen, "A tunable carbon nanotube electromechanical oscillator", Nature, vol. 431, pp. 284-287, 2004.

[20] Z. Ning, T. Shi, M. Fu, Y. Guo, X. Wei, S. Gao, et al., "Transversally and Axially Tunable Carbon Nanotube Resonators In Situ Fabricated and Studied Inside a Scanning Electron Microscope", Nano Lett., vol. 14, pp. 1221-1227, 2014.

[21] Z. Ning, M. Fu, G. Wu, C. Qiu, J. Shu, Y. Guo, et al., "Remarkable influence of slack on the vibration of a singlewalled carbon nanotube resonator", Nanoscale, vol. 8, pp. 8658-8665, 2016.

[22] H. Üstünel, D. Roundy, and T. Arias, "Modeling a suspended nanotube oscillator", Nano Lett., vol. 5, pp. 523$526,2005$.

[23] M. Tomi, A. Isacsson, M. Oksanen, D. Lyashenko, J.-P. Kaikkonen, S. Tervakangas, et al., "Buckled diamond-like carbon nanomechanical resonators", Nanoscale, vol. 7, pp. 14747-14751, 2015.

[24] S. Truax, S.-W. Lee, M. Muoth, and C. Hierold, "Axially tunable carbon nanotube resonators using co-integrated microactuators", Nano Lett., vol. 14, pp. 6092-6096, 2014.

[25] M. I. Younis, MEMS linear and nonlinear statics and dynamics vol. 20: Springer Science \& Business Media, 2011. 
[26] N. Kacem and S. Hentz, "Bifurcation topology tuning of a mixed behavior in nonlinear micromechanical resonators", Appl. Phys. Lett., vol. 95, pp. 183104, 2009.

[27] I. V. Antonova, V. P. Popov, J. Bak-Misiuk, J. Domagala, A. Misiuk, V. I. Obodnikov, A. K. Gutakovskii, A. RomanoRodriguez, "Effect of stress on defect transformation in hydrogen implanted silicon and SOI structures", Proc. SPIE, vol. 4412, pp. 120-125, 2001.

[28] F. D. Bannon, J. R. Clark, and C.-C. Nguyen, "High-Q HF microelectromechanical filters", IEEE J. Solid-state Circ., vol. 35, pp. 512-526, 2000.

[29] C. T.-C. Nguyen, "MEMS technology for timing and frequency control", IEEE Trans. Ultra., Ferro. And Freq. Control, vol. 54, 2007.

[30] K. Wang, A.-C. Wong, and C. T.-C. Nguyen, "VHF freefree beam high-Q micromechanical resonators", $J$. Microelectromech. Syst., vol. 9, pp. 347-360, 2000.

[31] X. M. H. Huang, C. A. Zorman, M. Mehregany, and M. L. Roukes, "Nanoelectromechanical systems: Nanodevice motion at microwave frequencies", Nature, vol. 421, pp. 496-496, 2003.

[32] J. E. E. Zekry "Microsystems encapsulation using nanoporous alumina", $\mathrm{PhD}$ Thesis, page 9, 2014.

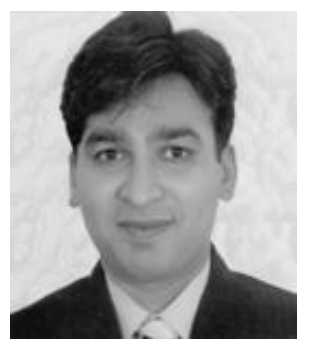

Syed N. R. Kazmi received M.Sc. degree in Physics from the Department of Physics, Quaid-I-Azam University, Islamabad, Pakistan, in 2001. In 2014, he received his $\mathrm{PhD}$ degree in Electrical Engineering from University of Twente, The Netherlands. From March 2013 till May 2015, he served as a Senior Scientist at National Institute of Lasers and Optronics (NILOP), Islamabad, Pakistan. He is currently working as a Post-doctoral fellow at King Abdullah University of Science Technology, Thuwal, Kingdom of Saudi Arabia. His research interests include materials deposition and characterization for NEMS/MEMS resonators, Nano/Micro device fabrication, and 2D material based resonators.

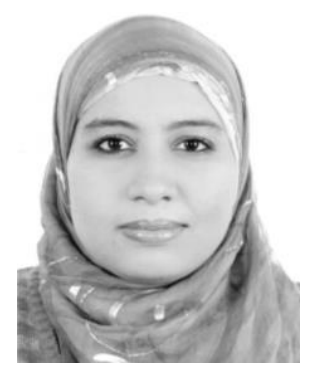

Amal Z. Hajjaj received the B.S. and master's degrees in mechanical engineering from Tunisia Polytechnic School in 2012 and 2013, respectively. She is currently pursuing the Ph.D. degree in mechanical engineering with the King Abdullah University of Science and Technology, Thuwal, Saudi Arabia. She is interested in characterizing theoretically and experimentally the linear and nonlinear dynamics of MEMS-based resonators with their applications in MEMS sensors and actuators.

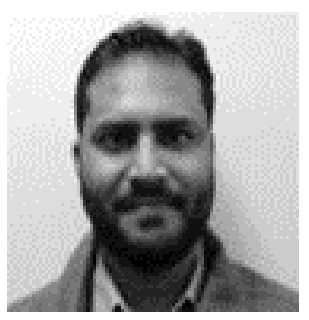

Md A. Al Hafiz received B.Sc. degree in Electrical and Electronic Engineering from the Islamic University of Technology (IUT), Dhaka, Bangladesh, in 2001. From April 2003 to February 2006, he served as a lecturer in the Department of EEE at the Rajshahi University of Engineering and Technology (RUET), Rajshahi, Bangladesh. He received his $\mathrm{Ph} . \mathrm{D}$. degree in Electrical Engineering from the University of New South Wales (UNSW), Sydney, Australia in 2011. He is currently working as Post-doc Fellow at KAUST. His research interests are MEMS/MOEMS systems, micro/nano-resonators, micro/nano-fabrication, sensors, actuators, planar lightwave technologies, optical interconnects, and optical cross-connect switches.

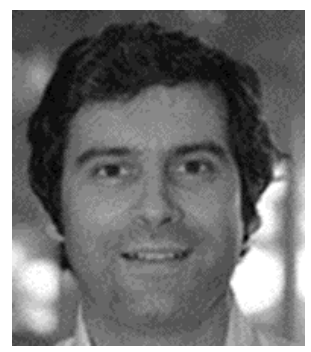

Pedro M. F. J. Costa is an Assistant Professor at the Materials Science and Engineering Program of King Abdullah University of Science and Technology (KAUST), Saudi Arabia. He leads the Laboratory for Carbon Nanostructures KAUST and also holds an Adjunct Assistant Professor position at the University of Aveiro (UA), Portugal.

Previously, he was a Junior Group Leader at UA and a Visiting Researcher at the IFW-Dresden, Germany. He (co-)authored numerous communications in matters related to carbon nanostructures, electron microscopy and semiconductors. He received several awards such as the Japan Carbon Award for Young Researchers and an Alexander von Humboldt Fellowship for Experienced Researcher.

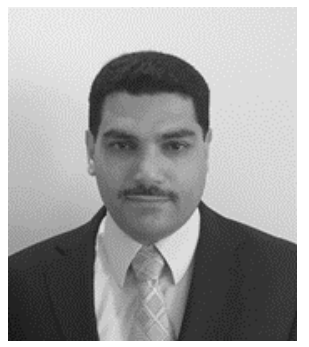

Mohammad I. Younis received the $\mathrm{Ph} . \mathrm{D}$. degree in engineering mechanics from Virginia Polytechnic Institute and State University, Blacksburg, VA, USA, in 2004. He is currently an Associate Professor of Mechanical Engineering with the King Abdullah University of Science and Technology, Thuwal, Saudi Arabia, and the State University of New York (SUNY), Binghamton, NY, USA. He serves as the Director of the MEMS and NEMS Characterization and Motion Laboratory. Dr. Younis is a recipient of the SUNY Chancellor's Award for Excellence in Scholarship and Creative Activities in 2012, the National Science Foundation Faculty Early Career Development Award in 2009, and the Paul E. Torgersen Graduate Research Excellence Award in 2002. He holds several U.S. patents in MEMS sensors and actuators. He serves as an Associate Editor of Nonlinear Dynamics, the Journal of Computational and Nonlinear Dynamics, and the Journal of Vibration and Control. He has authored the book entitled MEMS Linear and Nonlinear Statics and Dynamics (Springer, 2011). He is a member of the American Society of Mechanical Engineers and IEEE. 